\title{
An assessment of dietary intake, food avoidance and food beliefs in patients with ulcerative colitis of different disease status
}

\author{
Nor Hamizah Shafiee ${ }^{1}$, Zahara Abdul Manaf ${ }^{1}$, Norfilza M. Mokhtar ${ }^{2,3}$, Raja Affendi Raja Ali ${ }^{3,4}$ \\ ${ }^{I}$ Dietetic Unit \& Centre of Healthy Ageing and Wellness, Faculty of Health Sciences and ${ }^{2}$ Department of Physiology, Faculty of Medicine, \\ National University of Malaysia, Kuala Lumpur; ${ }^{3}$ GUT Research Group, Faculty of Medicine, National University of Malaysia, Kuala Lumpur; \\ ${ }^{4}$ Gastroenterology Unit, Department of Medicine, UKM Medical Centre, Kuala Lumpur, Malaysia
}

Background/Aims: Ulcerative colitis (UC) is a chronic, relapsing and remitting inflammation of the gastrointestinal tract. Little is known about the link between dietary intake, food avoidance, and beliefs among UC patients of different disease severity. Therefore, this study aimed to assess the dietary intake, food avoidance, and beliefs among active and inactive UC patients. Methods: A cross-sectional study was conducted among UC patients from a tertiary medical center in Kuala Lumpur, Malaysia. Demographic, anthropometric, dietary intake, food avoidance and beliefs were assessed. Disease activity of UC patients was evaluated using the Powell Tuck Index. Results: UC patients were recruited (64.1\% inactive UC and 35.9\% active UC). As compared to inactive UC patients, active UC patients were likely to lose weight ( $75.0 \%$ vs. $0 \%$ ), possess certain food beliefs (95.7\% vs. $39.0 \%)$, and frequently practiced dietary avoidance (95.7\% vs. $43.9 \%)$. The dietary intake among inactive UC patients was higher than active UC patients. However, neither of them met the standard nutrients recommendation for protein, calcium, iron, folate, zinc, vitamin D, vitamin $\mathrm{B}_{12}$, and vitamin E. Conclusions: Active UC patients had poorer dietary intake, were more prone to practicing food avoidance and exhibited certain food beliefs as compared to inactive UC patients. Both macro- and micronutrients intakes were inadequate regardless of patient's disease status. These findings emphasized the importance for patients to be provided with the nutrition-related knowledge as part of strategies to avoid nutritional inadequacies. (Intest Res 2020;18:447-458)

Key Words: Ulcerative, colitis; Foods; Diet; Nutrients intake

\section{INTRODUCTION}

Ulcerative colitis (UC) is chronic, relapsing and remitting inflammation of the gastrointestinal (GI) tract characterized by

Received April 15, 2019. Revised March 27, 2020. Accepted March 28, 2020 Correspondence to Zahara Abdul Manaf, Dietetic Unit \& Centre of Healthy Ageing and Wellness, Faculty of Health Sciences, National University of Malaysia, Jalan Raja Muda Abdul Aziz, Kuala Lumpur 50300, Malaysia. Tel: +60-3-92897677, Fax: +60-3-26947621, E-mail: zaharamanaf@ukm.edu.my Co-Correspondence to Raja Affendi Raja Ali, Gastroenterology Unit, Department of Medicine, UKM Medical Centre, Clinical Block Jalan Yaacob Latif, Bandar Tun Razak, Cheras, Kuala Lumpur 56000, Malaysia. Tel: +60-391456094, Fax:+60-3-91456692, E-mail: draffendi@ppukm.ukm.edu.my clinical symptoms such as abdominal pain, bloody diarrhea, poor appetite, nausea and vomiting, which can affect the dietary intake and nutrients absorption. ${ }^{1}$ The incidence and prevalence of UC are increasing globally and there appears to be a marked ethnic variation in the incidence of UC. ${ }^{2}$ To date, the precise pathophysiology underlying UC remains unclear $^{3}$ and the natural history of the disease includes the period of alternating active and inactive state. ${ }^{4}$ Disruption of the GI tract due to inflammation may lead to many nutritional complications particularly those related to a decrease in dietary intake, avoidance of eating, malabsorption, and nutrients inadequacies. ${ }^{5}$

Malnutrition is a common problem among patients with in- 
flammatory bowel disease (IBD); and up to $18 \%-62 \%$ of UC patients were reported to be malnourished. ${ }^{6}$ However, there is still insufficient data available to provide dietary recommendations in these patients on dietary modifications ${ }^{7}$ since there is no specific diet available for UC patients of different disease status and severity. Such limitations can lead to a change in the nutritional requirements and dietary patterns among these patients. It is also not uncommon for UC patients to avoid certain types of food items and possess certain food beliefs due to fear of having flares of disease that is associated with specific symptoms such as abdominal pain and diarrhea. ${ }^{7.8}$

Dietary restrictions involving frequent avoidance of certain food groups as practiced by both active and inactive UC patients may lead to nutritional complications. ${ }^{8,9}$ Anemia is a frequent complication related to UC patients and it is mainly due to the ongoing blood loss as a result of ulceration in the digestive tract lining ${ }^{10}$ and due to inadequate intake of iron, folate and vitamin $\mathrm{B}_{12}{ }^{11}{ }^{11}$ It is observed that among UC patients, restriction in dietary intake of both calcium and vitamin $\mathrm{D}$ is mainly due to limited intake of dairy products. ${ }^{12}$ This may eventually lead to an increase in the risk of developing osteoporosis among patients with UC. Besides, dietary intake of UC patients can also be compromised due to the heightened disease activity and complications related to the disease process. ${ }^{11}$

Dietary modifications are options for clinicians as well as patients in the management of IBD to control disease activity and symptoms. ${ }^{13}$ However, little is known about the effects of the disease activity on the dietary intake and practices of food avoidance among UC patients. Although a few published studies reported on the nutritional status of patients with $\mathrm{UC}^{8,14}$ none had reported on food intake and food avoidance practices. There are however a few studies available on the dietary beliefs and practices among IBD patients. ${ }^{15-17}$ To the best of our knowledge, this is the first study conducted in Malaysia to assess the nutritional intake of UC patients according to disease states and food avoidance practices. Findings from this study can be used in formulating nutritional guidelines for UC patients in the future.

\section{METHODS}

\section{Study Design and Patients}

A cross-sectional study using purposive sampling was conducted at the gastroenterology clinics in a tertiary medical center in Kuala Lumpur, Malaysia. Based on the sample size calculation, ${ }^{18} 64$ patients were required to provide $80 \%$ of power at a significant level of $5 \%$. The sample size was calculated based on the total number of 142 patients diagnosed with UC registered in the Universiti Kebangsaan Malaysia Medical Center IBD registry. Eligibility criteria for this study were patients of more than 18 years of age with confirmed UC, and they have no personal or family history of celiac disease, malabsorption disorders or GI malignancies and no previous bowel resections. Diabetic patients or those with a severe internal medical condition such as renal disease, lactose or gluten intolerance who required dietary restriction were excluded. Those who were pregnant or breastfeeding were also excluded from this study. Patients who attended the clinics were approached and identified for eligibility. Those who met the criteria were given an information sheet and written informed consent was obtained from all the patients. Ethical approval for this study was obtained from the Universiti Kebangsaan Malaysia Research Ethics Committee (approval No. NN-2017-133).

\section{Data Collection}

A semi-structured modified questionnaire was administered by a research dietitian to 64 patients through a one-to-one interview to collect data regarding demographic details, food intake, food avoidances and food beliefs between January to December 2018. The interview was conducted at the outpatient clinic, while patients waited for clinicians' review. Anthropometric measurements were taken before proceeding with the interview session. The duration taken for the interview was within an hour per patient.

\section{Dietary Assessment}

Dietary intake was assessed retrospectively using the validated Dietary History Questionnaire (DHQ) ${ }^{19}$ that was developed to obtain the information on food intake and usual dietary habits. In our study, most of the patients had a regular follow-up every 6 months, thus, dietary assessment using DHQ was taken for that period of time to assess the long-term habitual dietary intake among patients. Seven days weighing record method was used to assess the relative validity of the DHQ as this technique is considered as the "gold standard" to assess the dietary intake, providing that the subjects do not change their dietary habits. ${ }^{20}$ The design of this DHQ was based on the local dietary habits of the Malaysian population. The DHQ was formatted and included the detailed instructions and guidelines for the interviewer so that the information on the usual intake could be obtained using non-leading and open- 
ended questions.

The food items listed in the DHQ were categorized into 7 food groups namely, cereal and cereal products including cooked rice and noodles, traditional snacks, meat, poultry, fish, internal organs and their products, vegetables and fruits, milk and dairy products, water and soft drinks, and condiments/snacks. The traditional regional Malaysian foods in the DHQ was different compared to the Western-based food pattern. Generally, Western-based food pattern contains a significantly higher amount of red meat and processed meat, high-fat dairy products, fast foods, refined grains, and high sugar drinks and desserts, and a relatively lower amount of fruits, vegetables, wholegrain foods, fish and poultry. ${ }^{21}$

To minimize the errors while assessing the dietary intake of patients, the interview process was started by asking the patient to recall all the food items usually consumed daily and the recall process was built up for the period of weeks and months. Patients were also asked where, when and with whom each meal was eaten. For the specific food items mentioned, questions regarding types, portion size, and cooking methods used were needed for detail clarifications of regular dietary intake during the last 6 months. If the patient normally consumed a specific food item, how much he or she eats must be specifically noted either per day, per week or per month. Household measures available in the "Atlas of food exchanges and portion sizes" ${ }^{\prime 2}$ such as bowls, spoons, cups, and matchbox sizes were used to enhance the portion size estimation.

The frequencies of the food taken in the DHQ was then been converted into the amount of food intake using the devised formula by Wessex Institute of Public Health University of Southampton, 1995 and; the formula is given in the following equation. ${ }^{23}$

Weight of the food (g) per day= frequency of food intake (conversion factor $) \times$ serving size $\times$ number of serving $\times$ weight of food in one serving (g).

The 4th edition of nutrient composition of Malaysian foods ${ }^{24}$ was also used as a reference for the accurate analysis of the data. From the values of the amount of food per day, the detailed analysis intake of nutrients was calculated using the Nutritionist Pro ${ }^{\mathrm{TM}}$ Diet Analysis software (Axxya Systems, Woodinville, WA, USA). The percentage intake from recommendation was calculated by comparing the observed individual's intakes against the standard recommended nutrient intake for Malaysia (reference nutrient intake [RNI], 2017). ${ }^{25}$

\section{Questionnaire of Dietary Practices and Beliefs}

A semi-structured modified questionnaire was drafted after a careful review of the literature. This questionnaire was divided into three sections namely, sociodemographic data of patients, food beliefs, and food avoidance. The patient's dietary beliefs questionnaire was adapted and modified based on a previous survey of people with IBD about food view and nutrition. ${ }^{26}$ This questionnaire was modified by eliminating the unrelated questions, specifically those questions related to Crohn's disease. For this section, patients were asked to state as many as possible regarding their main beliefs on food and nutrition and sources of nutritional information. The main beliefs on food and nutrition included the effects of specific food items on their symptoms, nutritional balance of diet, and body appearance. In this study, the nutritional balance of diet is defined as patient's concern that their daily diet must include both nutritionally and evenly a variety of food consisting of several food groups such as cereals, protein-based products, fruits, and vegetables. Body appearance/image is defined as self-perception of weight, body shape, or body mass index (BMI). Questions on the patient's view about the importance of diet in controlling the symptoms were also included.

Food avoidance practices were assessed using a modified questionnaire from the previous study conducted among adults with IBD. ${ }^{8}$ In our study, the questionnaire was modified in terms of the food items listed to suit the Malaysian population using the reference from nutrient composition of Malaysian foods. ${ }^{24}$ Patients were asked to indicate whether they avoided the listed food item and the primary reasons for avoiding these foods. The response scale is a 5-point Likert scale ranging from (1) Eating this food causes me to have GI upset with symptoms that last up to 24 hours; (2) Eating this food causes me to have GI upset with symptoms that last days to weeks; (3) I have $\mathrm{read} /$ heard that people with UC should avoid this food; (4) A health professional (doctor, nurse, dietitian, other) has advised me to avoid this food; and (5) I do not like this food (personal preference).

These questionnaires were translated to the Malay language and pilot tested for the clarity and relevance among $10 \mathrm{UC}$ patients. Minor amendments were made as needed. The reliability of the questionnaires was assessed, and Cronbach $\alpha$ coefficients showed a high reliability of 0.82 for food beliefs and 0.84 for food avoidances.

\section{Disease Activity Index}

The disease activity index was quantified retrospectively during the consultation and examination with a clinician based on the information obtained from the patients. The clinical dis- 
ease activity index of UC patients was assessed using the scoring system of Powell Tuck Index (PTI) using the 4-point Likert scale. ${ }^{27}$ In this study, PTI was used as the established index for the evaluation of disease activity of UC as it is a more feasible and easily calculated index of disease activity. The evaluation of disease activity using PTI include symptoms, physical signs, and sigmoidoscopic appearance within 1 month.

Patients with scores $\leq 3$ were categorized as being in remission state, scores between $4-10$ as mildly active disease, scores 11-14 as moderate activity and scores $\geq 14$ were those with the clinically severe activity of the disease. For the purpose of this study, we grouped the patients into 2 categories of the disease activity based on the references from previous studies. ${ }^{7.8}$ Patients with the inactive disease were those who reported being in a remission state or having minimal symptoms. Patients with active disease activity were those who reported having a mild, moderate and severe state with the total scoring of PTI more than 3 points.

\section{Anthropometric Measurements}

Anthropometric measurements of weight, height, and body fat percentage were performed before proceeding with the interview session. Weight and body fat percentage (\%) were measured by using the Tanita Body Composition Analyzer Model SC-330 (Tanita, Tokyo, Japan). Weight and body fat percentage were taken to the nearest $0.1 \mathrm{~kg}$ and $0.1 \%$, respectively. Normal body fat percentage for men and women was classified according to clinical practice guideline on obesity (2004), which is less than $25 \%$ and $35 \%$, respectively. ${ }^{28}$ The displayed BMI was classified into 3 different categories of BMI, which are underweight, normal body weight, overweight and obese. Patients with BMI $\geq 30 \mathrm{~kg} / \mathrm{m}^{2}$ are defined as obese whereas those with BMI $\geq 25 \mathrm{~kg} / \mathrm{m}^{2}$ are considered overweight according to the National Institute of Health expert panel on the identification, evaluation, and treatment of overweight and obese in adults that adopted the World Health Organization (WHO) classification for overweight and obesity. ${ }^{29}$ In this study, we grouped overweight and obese patients into one category of BMI. The measurements of a patient's height were recorded by using stadiometer Seca 213 (Seca, Hamburg, Germany). The previous weight of patients was taken from the medical record. The height and weight of every patient attending the clinic were measured before the consultation or examination by the clinician. A significant weight loss for IBD patients was defined as more than $5 \%$ loss of weight for the last 6 months. ${ }^{30}$

\section{Statistical Analysis}

Data were compiled and analyzed using IBM Products and Service Solution (SPSS) Statistics version 23 (IBM Corp., Armonk, NY, USA). The distribution of all the variables was checked for normality using skewness and kurtosis with verification using the Shapiro-Wilk test. No missing data was found in this study. Normally distributed data were described in mean and standard deviation whilst non-normally distributed data were presented in median (interquartile range). Descriptive analyses were used to describe the frequencies and proportion (\%) of all the quantitative data. Comparison of the sociodemographic, anthropometric, medical background and dietary beliefs variables between the active and inactive state of disease were analyzed using chi-square test or Fisher exact test for categorical data. Independent sample $t$-test or Mann-Whitney test were performed for comparison of the disease states and all the continuous variables. The significance level was set at $P<0.05$.

\section{RESULTS}

\section{Sociodemographic and Characteristics of Patients}

Sociodemographic and the detailed characteristics of patients are summarized in Table 1. A total of 64 UC patients were recruited, among which 41 patients (64.1\%) were categorized in the inactive state and 23 patients (35.9\%) were in the clinically active disease. The details regarding the proportion of UC patients according to the disease severity scores based on the PTI scoring system are shown in Fig. 1. Males (73.4\%) accounted for most participants in this study. The median age of patients in the active state and inactive state were 49 years (interquartile range [IQR], 31.0-57.0 years) and 53 years (IQR, 39.557.0 years) respectively. Analysis of the multi-ethnic participation showed a marked ethnic variation with Malay (75.0\%) accounting for most participants, followed by Chinese (17.2\%) and Indian (7.8\%) with no significant differences in the disease state $(P>0.05)$. Nearly two-thirds of the patients in our study had the tertiary level of education (70.3\%) and $81.2 \%$ were with employed status. Clinical evaluation of UC patients according to disease state is shown in Supplementary Table 1.

The mean BMI was $25.5 \pm 5.1 \mathrm{~kg} / \mathrm{m}^{2}$ with $54.7 \%$ being in the BMI categories of overweight and obese. No significant difference was found in the mean of body weight, BMI and percentage of body fat between the active and inactive disease $(P>0.05)$. Patients with active disease had significantly greater weight loss $(75.0 \%)$ while all the patients in the clinically inactive state 
Table 1. Sociodemographic and Characteristics of UC Patients According to Active and Inactive Disease State

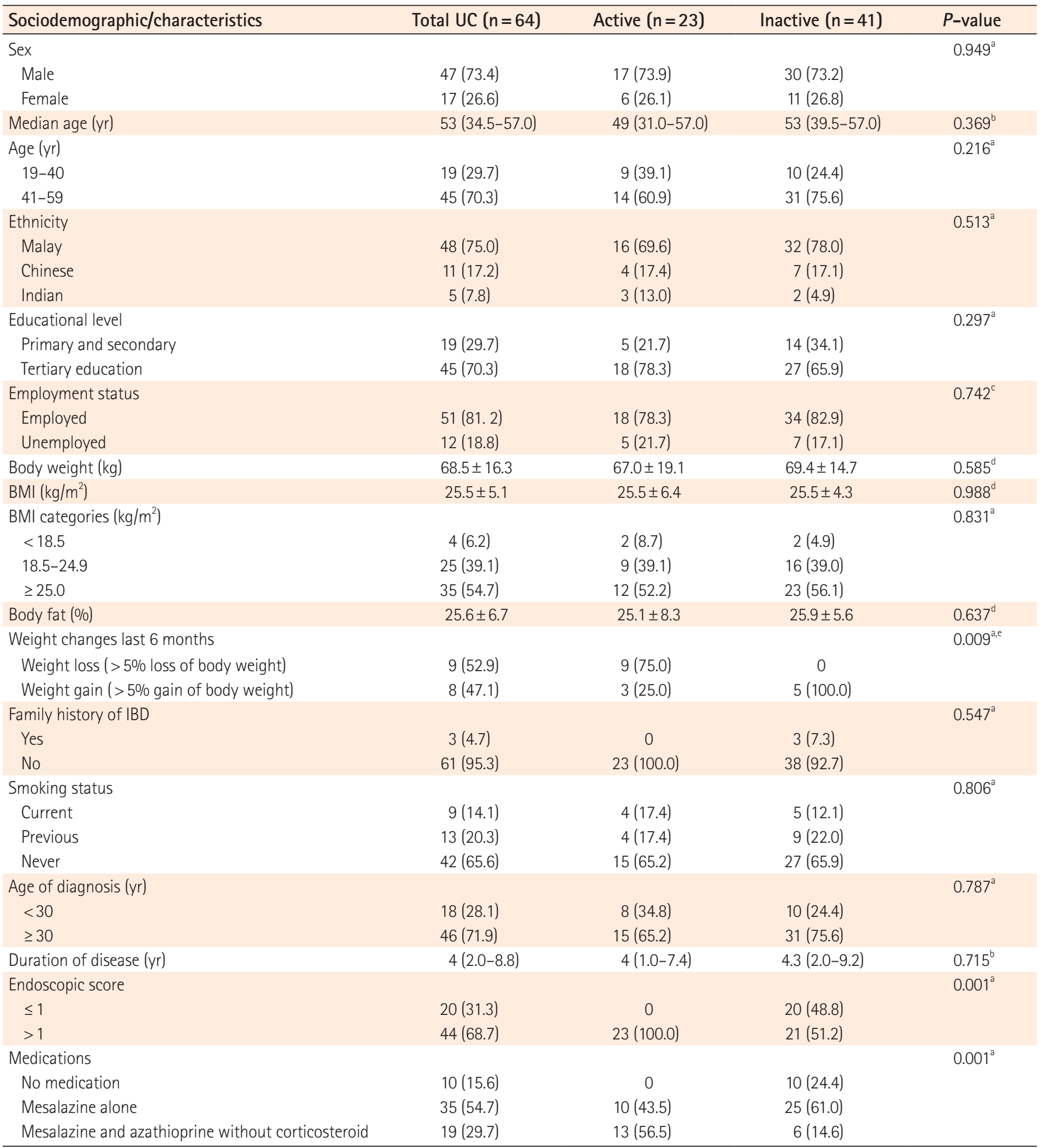

Values are presented as number (\%), median (interquartile range), or mean \pm standard deviation.

${ }^{a}$ Chi-square test.

${ }^{b}$ Mann-Whitney test.

'Fisher-exact test.

Independent sample $t$-test.

${ }^{e}$ According to Kondrup et al. (2003): Guidelines for Nutrition Screening 2002. ${ }^{30}$

UC, ulcerative colitis; BMI, body mass index; IBD, inflammatory bowel disease. 


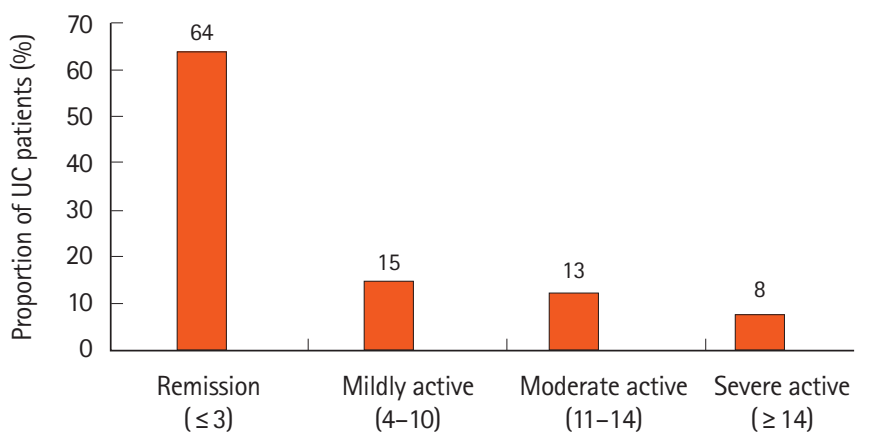

Fig. 1. Proportion of ulcerative colitis (UC) patients according to the disease severity scores based on Powell Tuck Index scoring system. had more weight gain in the last 6 months $(P<0.05)$ prior to the clinician's review. Only $4.7 \%$ had a family history of UC and $65.6 \%$ were non-smokers. The median disease duration among patients with UC in the active and inactive state was 4.0 years (IQR, 1.0-7.4 years) and 4.3 years (IQR, 2.0-9.2 years), respectively. Results showed that patients in the active state were significantly treated more with mesalazine and azathioprine without corticosteroids $56.5 \%$ whereas, patients in the inactive state had received predominantly mesalazine alone $(61.0 \%)(P<0.05)$.

Table 2. Comparison of Dietary Nutrient Intake of UC Patients According to the Active and Inactive Disease States

\begin{tabular}{|c|c|c|c|}
\hline \multirow{2}{*}{ Nutrients } & \multicolumn{2}{|c|}{ Intake, mean \pm SD (\% intake from recommendation) } & \multirow{2}{*}{$P$-value } \\
\hline & Active $(n=23)$ & Inactive $(n=41)$ & \\
\hline Energy (kcal) & $1,785 \pm 323.3(84.2)$ & $1,964 \pm 166.3(92.8)$ & $0.014^{\mathrm{a}, \mathrm{c}}$ \\
\hline Protein (g/kg BW) & $68.1 \pm 14.1(1.0 \mathrm{~g} / \mathrm{kg} \mathrm{BW})$ & $73.7 \pm 10.1(1.1 \mathrm{~g} / \mathrm{kg} \mathrm{BW})$ & $0.039^{\mathrm{a}, \mathrm{d}}$ \\
\hline Fat (g) & $66.5 \pm 17.9(33.4)$ & $72.4 \pm 10.0(34.2)$ & $0.457^{a, c}$ \\
\hline Carbohydrate (g) & $233.1 \pm 36.5(51.3)$ & $254.5 \pm 28.1(50.8)$ & $0.405^{a, c}$ \\
\hline Calcium (mg) & $482.6 \pm 200.0(46.3)$ & $542.7 \pm 201.1(59.7)$ & $0.039^{\mathrm{a}, \mathrm{c}}$ \\
\hline Iron (mg) & $9.8 \pm 2.0(65.1)$ & $11.8 \pm 1.8(82.9)$ & $0.041^{a, c}$ \\
\hline Folate $(\mu \mathrm{g})$ & $103.2 \pm 43.5(25.8)$ & $133.6 \pm 60.3(32.7)$ & $0.046^{\mathrm{a}, \mathrm{c}}$ \\
\hline Zinc $(\mathrm{mg})$ & $2.9 \pm 1.6(48.7)$ & $3.7 \pm 1.4(63.2)$ & $0.040^{\mathrm{a}, \mathrm{c}}$ \\
\hline Vitamin D $(\mu g)$ & $0.4 \pm 0.5(2.5)$ & $0.3 \pm 0.4(4.5)$ & $0.043^{b, c}$ \\
\hline Vitamin $B_{12}(\mu \mathrm{g})$ & $1.9 \pm 2.0(47.6)$ & $3.5 \pm 2.6(86.6)$ & $0.011^{b, c}$ \\
\hline Vitamin E (mg) & $3.1 \pm 2.0(34.6)$ & $3.7 \pm 2.7(41.7)$ & $0.300^{b, c}$ \\
\hline Vitamin C (mg) & $66.2 \pm 30.5(94.5)$ & $67.8 \pm 19.8(96.9)$ & $0.813^{a, c}$ \\
\hline
\end{tabular}

Independent sample t-test.

${ }^{b}$ Mann-Whitney test.

'According to Recommended Nutrient Intake for Malaysia, $2017 .{ }^{25}$

${ }^{d}$ According to European Society for Clinical Nutrition and Metabolism (ESPEN) Guidelines Clinical Nutrition for IBD $2017 .{ }^{31}$

UC, ulcerative colitis; SD, standard deviation; BW, body weight.

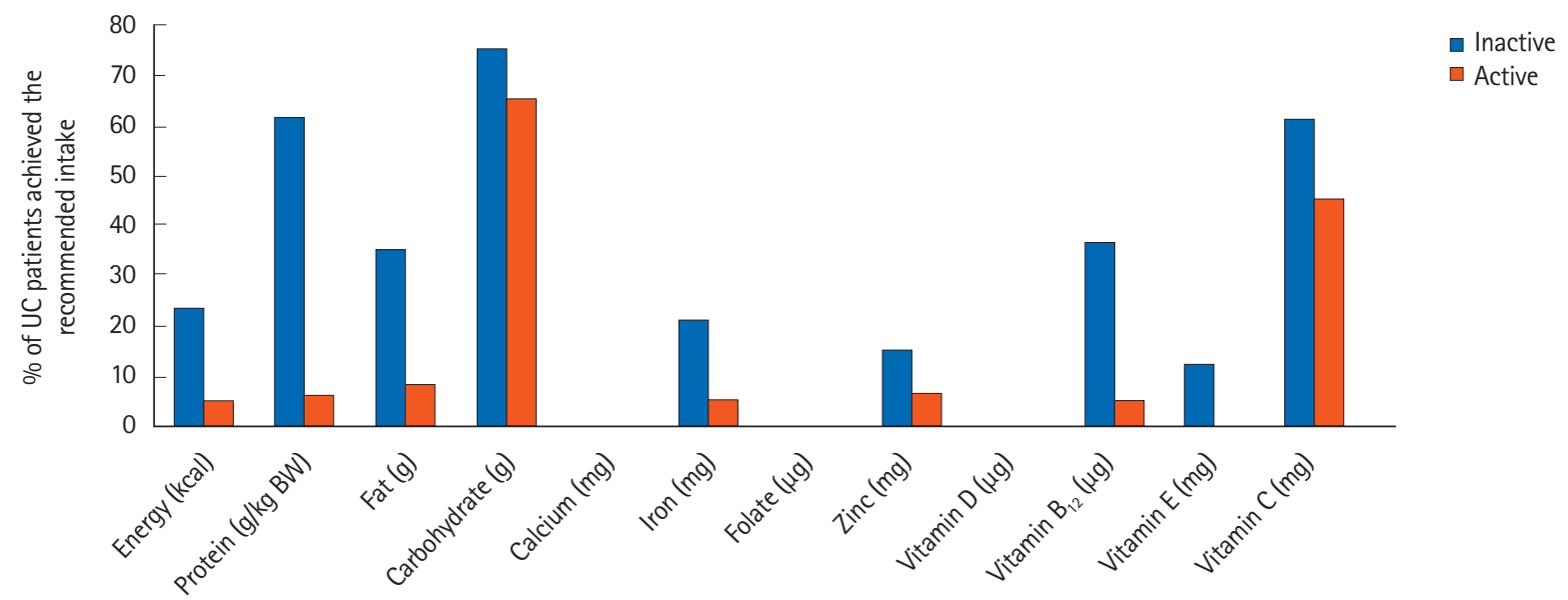

Fig. 2. Percentage of ulcerative colitis (UC) patients that achieved the recommended intake. BW, body weight. 


\section{Nutrients Intake of Patients According to the Disease Status}

The nutrients intake of patients was compared against the standard recommendation intake values according to the disease state as shown in Table 2. The dietary intake of patients in the inactive state was higher than the active state $(P<0.05)$, however, both groups of patients did not meet the standard recommendation for energy, protein, calcium, iron, folate, zinc, vitamin $\mathrm{D}$, vitamin $\mathrm{B}_{12}$, and vitamin $\mathrm{E}$. In particular, less than $10 \%$ of patients in the active disease met the recommendation for energy, protein, fat, calcium, iron, folate, zinc, vitamin D, vitamin $\mathrm{B}_{12}$, and vitamin $\mathrm{E}$ in comparison to the RNI for Malaysia 2017 as shown in Fig. 2.

\section{Food Avoidance of Patients According to the Disease Status}

Patients with clinically active disease significantly reported to practice more food avoidance (95.7\%) compared to patients in the inactive state of UC $43.9 \%(P<0.05)$. Food groups that are avoided by patients during the disease course of UC were cereals, nuts and seeds, processed food, milk and dairy products, red meat, sweetened beverages, legumes and oily foods as shown in Table 3. Generally, a higher proportion of patients always avoided processed food (35.9\%), followed by red meat (31.3\%), sweetened beverages (25.0\%), milk and dairy products (21.9\%), and oily food (18.8\%). Those in the clinically active state frequently reported avoiding taking milk/dairy products (28.1\%) and red meat (25.0\%) compared to those patients

Table 3. The Frequencies of Ulcerative Colitis Patients According to Food Avoidance Practices and Food Groups

\begin{tabular}{lcc}
\hline Food items & Always avoid & $\begin{array}{c}\text { Normally consume but avoid } \\
\text { when disease active }\end{array}$ \\
\hline Cereals (e.g., rice, bread, pasta) & $1(1.6)$ & $4(6.3)$ \\
Nuts and seeds & $5(7.8)$ & $1(1.6)$ \\
Processed food (e.g., sausage, nugget, French fries) & $23(35.9)$ & $6(9.4)$ \\
Milk and dairy products & $14(21.9)$ & $18(28.1)$ \\
Red meat & $20(31.3)$ & $16(25.0)$ \\
Sweetened beverages & $16(25.0)$ & $3(4.7)$ \\
Legumes (e.g., dahls, soybean, baked beans, soybean milk) & $9(14.1)$ & $7(10.9)$ \\
Oily food (e.g., deep-fried chicken/fish, fried traditional snacks) & $12(18.8)$ & $5(7.8)$ \\
\hline
\end{tabular}

Values are presented as number (\%).

Table 4. Reasons for Food Avoidance According to Food Groups among UC Patients

\begin{tabular}{|c|c|c|c|c|c|}
\hline \multirow[b]{2}{*}{ Food items } & \multicolumn{5}{|c|}{ Reasons option for avoidance ${ }^{a}$} \\
\hline & $\begin{array}{l}\text { Gl upset, } \\
24 \text { hours }\end{array}$ & $\begin{array}{c}\text { GI upset, } \\
\text { days to weeks }\end{array}$ & $\begin{array}{c}\text { Heard } \\
\text { "should avoid" }\end{array}$ & $\begin{array}{c}\text { Health professional } \\
\text { advice }\end{array}$ & $\begin{array}{l}\text { Personal } \\
\text { preference }\end{array}$ \\
\hline Nuts and seeds $(n=6)$ & $2(33.3)$ & $1(16.7)$ & $1(16.7)$ & 0 & $2(33.3)$ \\
\hline Processed food $(n=29)$ & $8(27.6)$ & 0 & $1(3.4)$ & $1(3.4)$ & $19(65.5)$ \\
\hline Red meat $(n=36)$ & $10(27.8)$ & $5(13.9)$ & $9(25.0)$ & $5(13.9)$ & $7(19.4)$ \\
\hline Sweetened beverages $(n=19)$ & $4(21.1)$ & 0 & $5(26.3)$ & $2(10.5)$ & $8(42.1)$ \\
\hline Legumes $(n=16)$ & $9(56.3)$ & 0 & $3(18.8)$ & 0 & $4(25.0)$ \\
\hline Oily food $(n=17)$ & $3(17.6)$ & 0 & $5(29.4)$ & $2(11.8)$ & $7(41.2)$ \\
\hline
\end{tabular}

Values are presented as number (\%).

${ }^{a}$ Reasons options for avoidance: (1) eating this food causes me to have Gl upset with symptoms that last up to 24 hours, (2) eating this food causes me to have GI upset with symptoms that last days to weeks, (3) I heard/read that people with UC should avoid this food, (4) a health professional has advised me to avoid this food, (5) I do not like this food.

UC, ulcerative colitis; Gl, gastrointestinal. 
Table 5. Frequencies of UC Patients According to Active and Inactive Disease States and Dietary Beliefs

\begin{tabular}{|c|c|c|c|c|}
\hline Nutritional concern & Total UC $(n=64)$ & Active $(n=23)$ & Inactive $(n=41)$ & $P$-value \\
\hline Concern/beliefs of food and nutrition & & & & $0.001^{\mathrm{a}}$ \\
\hline Affects the symptoms & $38(59.4)$ & $22(95.7)$ & $16(39.0)$ & \\
\hline Body appearance & $5(7.8)$ & $3(13.0)$ & $2(4.9)$ & \\
\hline Importance of diet in controlling the symptoms & & & & $0.001^{\mathrm{a}}$ \\
\hline Important & $41(64.1)$ & $22(95.7)$ & $19(46.3)$ & \\
\hline Nutritional sources of information used & & & & $0.675^{\mathrm{a}}$ \\
\hline Internet & $55(85.9)$ & $21(91.3)$ & $34(82.9)$ & \\
\hline Gastroenterologist & $55(85.9)$ & $21(91.3)$ & $34(82.9)$ & \\
\hline Dietitian & $1(1.6)$ & $1(4.3)$ & 0 & \\
\hline
\end{tabular}

Values are presented as number (\%).

${ }^{a}$ Chi-square test.

UC, ulcerative colitis.

in the inactive state. Based on results shown in Table 4, the primary reason for avoiding food items such as processed foods, sweetened beverages, and oily foods is due to personal preferences in which patients stated that they do not like this food. A large proportion of UC patients also reported that they avoided food items because they caused GI upset that lasted up to 24 hours (e.g., legumes 56.3\%, milk and dairy products $43.8 \%$, and red meat 27.8\%). Red meat, sweetened beverages, and oily foods were the food items most typically avoided after they read/heard that people living with UC should avoid taking these foods. Up to $13.9 \%$ of patients avoided red meat on the basis of health professional advice.

\section{Dietary Beliefs of Patients According to the Disease Status}

The dietary beliefs of patients in accordance with their disease states are summarized in Table 5. Patient's beliefs of food and nutrition were more frequently reported by patients in the active state of disease compared to those patients in the clinically inactive state $(P<0.05)$. Dietary beliefs reported were the effect on symptoms control (total $=38,59.4 \%$; active $95.7 \%$ and inactive $39.0 \%$ ), nutritional balance of diet (total $=30,46.9 \%$; active $73.9 \%$ and inactive $31.7 \%$ ) and body appearance (total $=5,7.8 \%$; active $13.0 \%$ and inactive $4.9 \%$ ). Among patients in clinically active disease, almost all of them (95.7\%) indicat- ed that diet is important in controlling the symptoms compared to the patients in the inactive disease $(P<0.05)$. Majority of the patients equally obtained their nutritional information from internet (85.9\%) and gastroenterologist (85.9\%), followed by family (59.4\%) and friends (39.1\%), whilst rarely from dietitians. Analysis demonstrated no significant association between nutritional source of information used in both active and inactive state of disease $(P>0.05)$.

\section{DISCUSSION}

In this study, we found that dietary intake was poorer among active UC patients as compared to inactive UC, possibly due to the lack of knowledge and awareness among patients, who are less certain about the role of diet on different disease severity whether the restriction or addition of specific food items might contribute to the increase of nutritional deficiencies. The most common causes of the nutrients inadequacies among UC patients were predominantly caused by the increased energy demand related to inflammation, impairment of nutrients absorption, and insufficient food intake due to the clinically active symptoms of the disease. ${ }^{6,31}$ Besides, food avoidance may also one of the contributing factors to poorer dietary intake among patients in the active state. ${ }^{15-17}$ However, in our study, overall we found that most of the "trigger foods" avoided 
were less than $30 \%$, which are similar with several studies that reported the avoidance of milk products and red meat was $29 \%$ and $27 \%$, respectively, ${ }^{8}$ whereas avoidance towards oily/ fatty foods and sweetened beverages accounted for $20 \%$ and $27 \%$, respectively. ${ }^{32}$

Although the data just showed a small percentage of UC patients who avoided the "possible harmful foods," our study found that the practice of food avoidances was more frequent in those with clinically active disease.

This is consistent with a recent Canadian study that showed significantly higher exclusion rates of foods during the active disease (39\%-69\%) compared to those patients in the inactive state. ${ }^{33}$ As patients frequently avoid certain food groups, patients might be at risk of insufficient dietary intake particularly those patients in the active state. Besides, our study also found that less than half of patients practiced food avoidance during the inactive disease, which is lower than findings from a previous study that demonstrated up to $71 \%$ of the patients with UC employed food avoidance practice during the inactive state of the disease.$^{34}$ Nevertheless, the current results showed that only a small pro- portion of patients $(<50 \%$ out of 41 patients) in this group achieved the nutrients intake recommendation

The current study demonstrated that patients with clinically active disease were more likely to exhibit beliefs that dietary intake was important in controlling the associated disease symptoms as compared to their counterparts in the inactive state. These findings are parallel with previous studies among IBD patients who also reported a decrease in dietary consumption of selected food items due to the active symptoms or fear of having a relapse in symptoms. ${ }^{6,7}$ In this study, patients sometimes avoid certain types of foods that they associate with disease activity, even they are uncertain that those foods elicit their symptoms. This might be related to the patient's lack of knowledge on foods to avoid and how to maintain their nutritional balance. Thus, appropriate nutritional interventions are needed to provide adequate information on dietary beliefs and nutritional outcomes among UC patients. Furthermore, prospective and comparative controlled study design should be carried out to make recommendations on the role of diet during the course of the disease by identifying and manipulating the "trigger foods" as the therapeutic strategies to manage and alleviate the symptoms.

The general recommendation for energy requirement for IBD patients is similar to those healthy populations as patients with IBD do not have increased energy expenditure as a result of the disease activity. ${ }^{31}$ However, we observed that almost all the patients did not meet the recommended energy intake, which may lead to weight loss particularly for those in the clinically active disease. The reported mean intake of macronutrients was comparable with the recommended nutrient intake for Malaysia, 2017 (RNI 2017). However, the mean intake of protein during active disease was less than $1.2-1.5 \mathrm{~g} / \mathrm{kg} /$ day as compared to European Society for Clinical Nutrition and Metabolism (ESPEN) guidelines clinical nutrition for IBD $2017^{31}$

The systemic inflammation during active disease increases the protein requirement, therefore, patients may consider supplementing with oral nutritional therapy if they cannot meet the protein requirements from normal food.

In our study, inadequacies of micronutrients in the clinically active disease were greater for the calcium, iron, folate, zinc, vitamin $B_{12}$, vitamin $D$, and vitamin $E$ in comparison with the UC patients in the inactive state. Similarly, a recent study showed the inadequate intake of vitamins as well as a deficiency in the in- take of the calcium, folate, and iron as a result of the decreased in the amount of food intake, lactose based products, fruits and vegetables, and meat. ${ }^{35}$ Patients in the active state were vulnerable to the nutritional inadequacies due to the chronically poor dietary intake associated with the long-lasting avoidance of particular food groups, nutrients loss from diarrhea and possible adverse effects of the disease treatments. ${ }^{25}$ Besides, long-term medication therapy could have an impact on the micronutrient absorption and utilization. ${ }^{6}$ Drugs administration could worsen the defective nutrient absorption. For example, aminosalicylates decrease folic acid absorption whereas corticosteroids can reduce the absorption of calcium from the GI tract and increase urine calcium excretion. ${ }^{32} \mathrm{In}$ our study, none of the enrolled UC patients were currently treated with steroid therapies.

More than half (54.7\%) of the patients in this study were over- weight and obese, indicating the coexistence of malnutrition and obesity. There is a risk of nutritional deficiencies even if the patients were overweight or obese. A higher BMI among the enrolled patients may be due to a sedentary lifestyle which is a predisposing factor for excess weight gain. Patients with UC were more likely to have a reduction in their activity level after IBD diagnosis. ${ }^{36}$ The use of corticosteroid therapy can contribute to the changes in body composition among IBD patients for a considerable period of time. A 12week randomized trial on the effects of corticosteroid towards the body composition in IBD patients suggested that corticosteroids increased BMI and body fat percentage. ${ }^{37}$ Unfortu- 
nately, in our study, there is no available data regarding physical activity level and the previous use of steroids for the enrolled patients. Furthermore, the biological agents used to induce remission state of disease also has been shown to result in an increased BMI and may be a contributing factor to rising rates of obesity in IBD patients. ${ }^{38,39}$ However, in our study, none of the enrolled patients was on these biological therapies.

In this study, most patients sought after nutritional information from a gastroenterologist and the internet in managing the nutrition-related complications due to UC.

However, the dietary information found on the internet or press may be unreliable and need further evaluation. The evidence-based dietary recommendations for UC are still lacking thus, it may lead patients with strong concerns regarding their disease to seek dietary advice from various sources. ${ }^{40}$ In this study, patients also preferred to ask their clinicians for the dietary recommendation in helping them to cope with the disease. Based on a previous study with 400 IBD patients, onethird of patients received nutritional advice from the dietitian, one-third from clinicians and only $15 \%$ sought information from the internet. ${ }^{15}$ This contradicts with our study, whereby only 1 out of 64 patients sought after nutritional advice from the dietitian. The results indicated that the behavioral influences of patients are unlikely to be affected by health professional advice. Nonetheless, our study did not further explore the reasons resulting in few patients obtaining nutrition information from dietitians.

Our study has several limitations. First, this study was crosssectional in nature therefore the result could not establish a true cause and effect of dietary avoidance and disease state. Second, this study was based on the questionnaire response, thus it might be biased in terms of the self-reported answers. Third, this study involved a selected group of patients with UC and most of them were in the inactive state of disease (64.1\%). It would have been advantageous to select more patients in active disease to further address the differences be- tween disease status regarding nutrients intakes and dietary practices. Biochemical indicators for nutritional status data such as hemoglobin, ferritin, serum vitamin D, and albumin were not included as these data were not recently available (at the time of interview) for most patients. Because of this, our data cannot determine the presence or absence of complications such as anemia and osteoporosis for these patients. In this study, we also do not have the details on information regarding the comorbid conditions of enrolled patients in our study although the comorbid conditions (e.g., hepatic disease, GI comorbidity, and cardiovascular disease) were frequently associated with their clinical course, and may change the dietary practices and medical management. Our study also does not have data regarding the extent of UC involvement although it would be advantageous to analyze the data according to the extent of UC involvement as it is an important predictor of disease prognosis and medical response.

In conclusion, active UC patients demonstrated poorer dietary intake and practiced more food avoidance and beliefs as compared to inactive UC patients. Nutritional deficiencies in UC patients, especially during flares of disease underpin the need for the trained dietitian to provide better nutrition education regarding the roles of nutrition in the management of IBD. Individualized dietary counseling for the food choices and other alternative nutritional supplements could be advocated to ensure adequate nutrients intake to these patients. Although the general dietary recommendation for UC patients is still lacking, our study had identified the specific food items that are frequently avoided by patients, which may negatively influence nutrition adequacies during the clinical course of the disease. Thus, the dietary counseling for the UC patients should be prioritized on the nutrient-dense foods, optimizing the dietary diversity as well as modifications on specific nutrients based on their disease activity.

\section{FINANCIAL SUPPORT}

The authors received no financial support for the research, authorship, and/or publication of this article.

\section{CONFLICT OF INTEREST}

No potential conflict of interest relevant to this article was reported.

\section{AUTHOR CONTRIBUTION}

Conceptualization and methodology: Shafiee NH, Manaf ZA, Raja Ali RA, Mokhtar NM. Writing-original draft and visualization: Shafiee NH. Writing-review and editing: ManafZA, Raja Ali RA. Approval of final manuscripts: all authors.

\section{ORCID}

Shafiee NH

https://orcid.org/0000-0002-5233-1384

Manaf ZA 


\author{
Mokhtar NM \\ https://orcid.org/0000-0002-4863-100X \\ Raja Ali RA \\ https://orcid.org/0000-0003-4995-3868
}

\section{ACKNOWLEDGMENTS}

We are grateful to Dr. Shanti Krishnasamy (National University of Malaysia, Kuala Lumpur, Malaysia) for her supervision during the preparation of the research proposal.

\section{SUPPLEMENTARY MATERIAL}

Supplementary materials are available at the Intestinal Research website (https://www.irjournal.org).

\section{REFERENCES}

1. Goyal N, Rana A, Ahlawat A, Bijjem KR, Kumar P. Animal models of inflammatory bowel disease: a review. Inflammopharmacology 2014;22:219-233.

2. Molodecky NA, Soon IS, Rabi DM, et al. Increasing incidence and prevalence of the inflammatory bowel diseases with time, based on systematic review. Gastroenterology 2012;142:4654 .

3. Ng WK, Wong SH, Ng SC. Changing epidemiological trends of inflammatory bowel disease in Asia. Intest Res 2016;14:111119.

4. Jakubowski A, Zagórowicz E, Kraszewska E, Bartnik W. Rising hospitalization rates for inflammatory bowel disease in Poland. Pol Arch Med Wewn 2014;124:180-190.

5. Wędrychowicz A, Zając A, Tomasik P. Advances in nutritional therapy in inflammatory bowel diseases: review. World J Gastroenterol 2016;22:1045-1066.

6. Scaldaferri F, Pizzoferrato M, Lopetuso LR, et al. Nutrition and IBD: malnutrition and/or sarcopenia? A practical guide. Gastroenterol Res Pract 2017;2017:8646495.

7. Cohen AB, Lee D, Long MD, et al. Dietary patterns and selfreported associations of diet with symptoms of inflammatory bowel disease. Dig Dis Sci 2013;58:1322-1328.

8. Vagianos K, Clara I, Carr R, et al. What are adults with inflammatory bowel disease (IBD) eating? A closer look at the dietary habits of a population-based Canadian IBD cohort. JPEN J Parenter Enteral Nutr 2016;40:405-411.

9. O'Sullivan M. Vitamin D as a novel therapy in inflammatory bowel disease: new hope or false dawn? Proc Nutr Soc 2015; 74:5-12.

10. Guagnozzi D, Lucendo AJ. Anemia in inflammatory bowel disease: a neglected issue with relevant effects. World J Gastroenterol 2014;20:3542-3551.

11. Silva AF, Schieferdecker ME, Amarante S. Food intake in patients with inflammatory bowel disease. ABCD Arq Bras Cir Dig 2011;24:204-209.

12. Brasil Lopes M, Rocha R, Castro Lyra A, et al. Restriction of dairy products; a reality in inflammatory bowel disease patients. Nutr Hosp 2014;29:575-581.

13. Lomer MC, Hart AL, Verjee A, Daly A, Solomon J, Mclaughlin J. What are the dietary treatment research priorities for inflammatory bowel disease? A short report based on a priority setting partnership with the James Lind Alliance. J Hum Nutr Diet 2017;30:709-713.

14. Walton M, Alaunyte I. Do patients living with ulcerative colitis adhere to healthy eating guidelines? A cross-sectional study. Br J Nutr 2014;112:1628-1635.

15. Limdi JK, Aggarwal D, McLaughlin JT. Dietary practices and beliefs in patients with inflammatory bowel disease. Inflamm Bowel Dis 2016;22:164-170.

16. de Vries JHM, Dijkhuizen M, Tap P, Witteman BJM. Patient's dietary beliefs and behaviours in inflammatory bowel disease. Dig Dis 2019;37:131-139.

17. Tomar SK, Kedia S, Upadhyay AD, et al. Impact of dietary beliefs and practices on patients with inflammatory bowel disease: an observational study from India. JGH Open 2017;1:1521.

18. Krejcie RV, Morgan DW. Determining sample size for research activities. Educ Psychol Meas 1970;30:607-610.

19. Shahar S, Earland J, Abdulrahman S. Validation of a dietary history questionnaire against a 7-D weighed record for estimating nutrient intake among rural elderly Malays. Malays J Nutr 2000;6:33-44.

20. Bingham SA, Nelson M, Paul AA, Haraldsdottir J, Loken EB, Van Staveren WA. Methods for data collection at an individual level. In: Cameron ME, Van Staveren WA, eds. Manual on methodology for food consumption studies. New York: Oxford University Press, 1988:53-59.

21. Barbaresko J, Koch M, Schulze MB, Nöthlings U. Dietary pattern analysis and biomarkers of low-grade inflammation: a systematic literature review. Nutr Rev 2013;71:511-527.

22. Suzana S, Noor Aini MY, Nik Shanita S, Rafidah G, Roslina A. Atlas of food exchanges and portion sizes. 2nd ed. Kuala Lumpur: MDC Publishers, 2009.

23. Norimah AK Jr, Safiah M, Jamal K, et al. Food consumption patterns: findings from the Malaysian Adult Nutrition Survey (MANS). Malays J Nutr 2008;14:25-39. 
24. Tee ES, Ismail MN, Nasir MA, Khatijah I. Nutrient composition of Malaysian foods. 4th ed. Kuala Lumpur: Institute for Medical Research, 1997.

25. National Coordinating Committee on Food and Nutrition. RNI Recommended Nutrient Intake for Malaysia: a report of the Technical Working Group on Nutritional Guideline. Putrajaya: Ministry of Health Malaysia, 2017.

26. Kinsey L, Burden S. A survey of people with inflammatory bowel disease to investigate their views of food and nutritional issues. Eur J Clin Nutr 2016;70:852-854.

27. Powell-Tuck J, Bown RL, Lennard-Jones JE. A comparison of oral prednisolone given as single or multiple daily doses for active proctocolitis. Scand J Gastroenterol 1978;13:833-837.

28. Ismail IS, Bebakar WM, Kamaruddin NA. Clinical practice guideline on management of obesity 2004. Putrajaya: Ministry of Health Malaysia, Academy of Medicine of Malaysia Malaysian Association for the Study of Obesity, Malaysian Endocrine and Metabolic Society, 2004.

29. Clinical guidelines on the identification, evaluation, and treatment of overweight and obesity in adults: the evidence report. National Institutes of Health. Obes Res 1998;6 Suppl 2:51S209S.

30. Kondrup J, Allison SP, Elia M, et al. ESPEN guidelines for nutrition screening 2002. Clin Nutr 2003;22:415-421.

31. Forbes A, Escher J, Hébuterne X, et al. ESPEN guideline: clinical nutrition in inflammatory bowel disease. Clin Nutr 2017; 36:321-347.

32. Marsh A, Kinneally J, Robertson T, Lord A, Young A, Radford Smith G. Food avoidance in outpatients with inflammatory bowel disease: who, what and why. Clin Nutr ESPEN 2019;31: $10-16$.

33. Bergeron F, Bouin M, D’Aoust L, Lemoyne M, Presse N. Food avoidance in patients with inflammatory bowel disease: what, when and who? Clin Nutr 2018;37:884-889.

34. Owczarek D, Rodacki T, Domagała-Rodacka R, Cibor D, Mach T. Diet and nutritional factors in inflammatory bowel diseases. World J Gastroenterol 2016;22:895-905.

35. Vidarsdottir JB, Johannsdottir SE, Thorsdottir I, Bjornsson E, Ramel A. A cross-sectional study on nutrient intake and -status in inflammatory bowel disease patients. Nutr J 2016;15:61.

36. Magee EA, Richardson CJ, Hughes R, Cummings JH. Contribution of dietary protein to sulfide production in the large intestine: an in vitro and a controlled feeding study in humans. Am J Clin Nutr 2000;72:1488-1494.

37. Nakahigashi M, Yamamoto T. Increases in body mass index during infliximab therapy in patients with Crohn's disease: an open label prospective study. Cytokine 2011;56:531-535.

38. Wiese D, Lashner B, Seidner D. Measurement of nutrition status in Crohn's disease patients receiving infliximab therapy. Nutr Clin Pract 2008;23:551-556.

39. Gatt K, Schembri J, Katsanos KH, et al. Inflammatory bowel disease [IBD] and physical activity: a study on the impact of diagnosis on the level of exercise amongst patients with IBD. J Crohns Colitis 2019;13:686-692.

40. Hou JK, Lee D, Lewis J. Diet and inflammatory bowel disease: review of patient-targeted recommendations. Clin Gastroenterol Hepatol 2014;12:1592-1600. 
See "An assessment of dietary intake, food avoidance and food beliefs in patients with ulcerative colitis of different disease status" on page 447-458.

Supplementary Table 1. Clinical Evaluation of Ulcerative Colitis Patients

\begin{tabular}{lcc}
\hline Clinical characteristics & Active disease & Inactive disease \\
\hline Bowel frequency (day) & 0 & $41(100.0)$ \\
$<3$ & $18(78.3)$ & 0 \\
$3-6$ & $5(21.7)$ & 0 \\
$\geq 6$ & & \\
Stools consistency & 0 & $20(48.8)$ \\
Formed & $8(34.8)$ & $21(51.2)$ \\
Semi-formed & $15(66.2)$ & 0 \\
Liquid & $23(100.0)$ & 0 \\
Abdominal pain & $23(100.0)$ & 0 \\
Anorexia & $10(43.5)$ & 0 \\
Nausea/vomiting & & 0 \\
Stools bleeding & $10(43.5)$ & 0 \\
Trace & 0 & 0 \\
More than trace & & 0 \\
Abdominal tenderness & $17(73.9)$ & 0 \\
Mild & $6(26.1)$ & 0 \\
Moderate & 0 & 0 \\
Severe & 0 & 0 \\
Rebound & & \\
\hline
\end{tabular}

Values are presented as number (\%). 